\title{
The development of simile comprehension: From similarity to scalar implicature
}

\author{
Madeleine Long, Vishakha Shukla and Paula Rubio-Fernández* \\ University of Oslo
}

Preschool children were tested on a new type of scalar implicature: saying 'Betty is like a nurse', normally implies that Betty is not a nurse. Experiment 1 used a guessing game with children 3, 4, 5, 6 and 13 years ( $\mathrm{N}=99)$, who were given metaphors ('Lucy is a parrot') or similes ('Lucy is like a parrot') as clues to select one of three drawings (a parrot, a girl or a parrot-looking girl).

Experiment 2 (ages 3, 4, 5 and adults; $\mathrm{N}=201$ ) focused on the simile condition, and Experiment 3 (ages 3, 4 and 5; N=48) confirmed a similarity baseline. The results show a developmental trend in the derivation of scalar implicatures during preschool, starting as early as 3 years.

Keywords: simile, metaphor, scalar implicature, scalar terms, pragmatic reasoning.

*Correspondence to: paula.rubio-fernandez@,ifikk.uio.no

\section{Acknowledgements}

This research was supported by a Young Research Talent Grant awarded to PRF (Research Council of Norway, 230718). The authors gratefully acknowledge this funding. 


\section{Introduction}

Children's ability to compare members of different categories has been studied in various areas of communicative development, ranging from early overextensions (e.g., referring to a horse as a 'doggy'; Clark, 1973; Bloom, 2002; Gershkoff-Stowe et al., 2006) to pretend play (e.g., playfully referring to a bucket as a hat; Winner et al., 1979, Hudson \& Nelson, 1984; Vosniadou, 1987a) and, centrally, to the comprehension of similes and metaphors (Vosniadou et al., 1984; Seidenberg \& Bernstein, 1986; Di Paola et al., 2019). Both similes and metaphors require comparing members of different categories (as in examples (a) and (b) below), whereas literal comparisons and categorization statements are applied within the same category (as in (c) and (d)):

a. Fred is like a lion.

b. Fred is a lion.

c. Fred is like his brother.

d. Fred is an engineer.

Studies on metaphor and simile comprehension report that for young children, similes are easier to interpret than metaphors, probably because the comparison is made explicit (Reynolds \& Ortony, 1980; Vosniadou \& Ortony, 1983; Vosniadou et al., 1984; Seidenberg \& Bernstein, 1986; Happé, 1995). Children's understanding of similes is related to the development of figurative language (Vosniadou \& Ortony, 1983) and analogies (Vosniadou, 1995), both of which require pragmatic reasoning: what is the intended ground of a simile or an analogy? There are, for example, different ways in which Fred could be like a lion: his hair may resemble a lion's mane, or he may be strong like a lion (Rubio-Fernandez \& Grassmann, 2016). Here we will propose that similes (or comparison statements, more generally) involve another form of 
pragmatic reasoning that has been overlooked in the literature: namely, the derivation of scalar implicatures (Rubio-Fernandez et al., 2017).

\section{Developmental pragmatics and scalar implicatures}

Scalar implicatures are a type of pragmatic inference whereby a speaker uses the weaker of two terms and the listener is entitled to assume that the stronger term does not apply. Consider the following examples:

e. Wilma ate some of the cookies in the jar.

f. Wilma ate all of the cookies in the jar.

If Wilma had eaten all of the cookies, a cooperative speaker should utter (f), from which it follows that if the same speaker chose to utter (e), that must mean Wilma did not eat all of the cookies. This, however, is a pragmatic inference and not part of the semantic meaning of 'some', which is compatible with 'all'. For example, if the speaker was not sure whether or not Wilma had eaten all of the cookies, they would utter (e), but in that situation, the listener would not be entitled to infer that the stronger statement did not apply.

There is an extensive experimental literature on children's derivation of scalar implicatures (for recent reviews, see Papafragou \& Skordos, 2016; Horowitz et al., 2018), which aims to tap into the emergence of the semantics/pragmatics distinction. In other words, when do children start deriving meaning beyond what is literally said and understand what is implied? In line with early studies in the 70s and 80s (e.g., Smith, 1980; Braine \& Rumain, 1981), Noveck (2001) observed that 8- and 10-year-old French-speaking children accepted statements such as 'Some elephants have trunks' as true, suggesting a logical interpretation of the quantifier (gloss: some and maybe all elephants have trunks). More recent studies have tried to overcome the limitations 
of earlier paradigms (e.g., the lack of context and motivation to derive a pragmatic interpretation), revealing an earlier sensitivity to pragmatic inference: under certain experimental conditions, children as young as 5 have been shown to derive scalar implicatures, although not at adult-like levels (e.g., Chierchia et al., 2001; Gualmini et al., 2001; Papafragou \& Musolino, 2003; Guasti et al., 2005; Pouscoulous et al., 2007; Katsos \& Bishop, 2011).

One argument that has been put forward to explain young children's limitations with scalar implicatures is the need to consider alternative expressions, which in turn requires learning the corresponding scale-mates (e.g., 'or' vs 'and': 'Barney will have cake or soda' vs 'Barney will have cake and soda'; 'may' vs 'have to': 'Dino may be in the house' vs 'Dino has to be in the house'). Supporting this hypothesis, recent studies have shown an increase in preschoolers' derivation of scalar implicatures when lexical alternatives are clearly contrasted (e.g., Miller et al., 2005; Barner et al., 2011; Skordos \& Papafragou, 2016). This is one factor that was manipulated in the present study in order to investigate preschoolers' derivation of scalar implicatures during simile interpretation.

\section{Comparison statements can license scalar implicatures}

Scalar terms giving rise to implicatures are characterized by a relation of unilateral entailment; that is, a sentence using the weaker term is true whenever the equivalent sentence using the stronger term is true, but not vice-versa. This is clear when comparing canonical examples with 'some' and 'all': whenever Wilma eats all the cookies, it is true that she ate some cookies. However, it is not always the case that when Wilma eats some cookies, she ate all the cookies. Rubio-Fernández et al. (2017) have recently argued that the same relationship holds between comparison and categorization statements: 
g. Betty is like a nurse.

h. Betty is a nurse.

Thus, if Betty is a nurse, she is surely similar to a nurse. However, if Betty was like a nurse, it would not necessarily hold that she was an actual nurse (for semantic diagnostics and experimental evidence with adults, see Rubio-Fernández et al., 2017).

The fact that comparison and categorization statements form a scale explains two phenomena frequently discussed in the figurative language literature. First, metaphors are perceived to be stronger or more expressive than their corresponding similes (e.g., 'Fred is a bulldozer' vs 'Fred is like a bulldozer'; Ortony, 1979; Zharikov \& Gentner, 2002; Glucksberg, 2011). Also, comparison statements seem infelicitous when the corresponding categorization statement holds (e.g., '*A mango is like a fruit' or '*A zebra is like an animal'; Glucksberg \& Keysar, 1990; Carston, 2002; Barnden, 2012). Rubio-Fernández et al. (2017) argue that, whereas it is logically true that a mango is like a fruit or a zebra is like an animal, these comparisons feel like understatements because mangoes are fruits (not just like fruits) and zebras are animals (not merely similar to animals).

While early studies have compared children's interpretation of similes and metaphors (Reynolds \& Ortony, 1980; Vosniadou \& Ortony, 1983; Vosniadou et al., 1984; Seidenberg \& Bernstein, 1986; Happé, 1995), this is the first study to investigate whether preschool children appreciate that similes and metaphors (or categorization and comparison statements, more generally) form a scale, which can be used to derive scalar implicatures.

\section{Do preschoolers derive scalar implicatures when interpreting similes?}


The main aim of the present study was to investigate preschoolers' ability to understand that statements of the form ' $x$ is like a $y$ ' may imply ' $x$ is not a $y$ ' (e.g., if someone says that Betty is like a nurse, they would normally imply that she is not a nurse). We hypothesize that the scale formed by categorization and comparison statements may be particularly accessible to preschool children because these two types of statement are probably highly frequent in child-directed speech (e.g., 'A whale is a mammal' or 'A clementine is like an orange'). It is therefore possible that an earlier sensitivity to pragmatic inference may be observed with these scalar expressions.

The secondary aim of the study was to test preschoolers' appreciation of the ambiguity between literal and figurative meanings. For example, taken out of context, the sentence 'Fred is a lion' could literally refer to a lion (with a person's name) or metaphorically to a person (with lion-like properties). Analogously, 'Fred is like a lion' could be a simile (figuratively comparing a person with an animal) or a literal comparison (between a specific lion and the lion kind). Experimental studies on the development of figurative language have not yet established at what age children become aware of these ambiguities (cf. Vosniadou \& Ortony, 1983).

In order to investigate these two questions, Experiment 1 used a 3-choice task (see also Stiller et al., 2015; Horowitz et al., 2018) embedded in the game 'I spy with my little eye', in which the Experimenter gave the child a clue either in the metaphor or simile form (e.g., 'Lucy is a parrot' or 'Lucy is like a parrot'; see Fig. 1) from which the child had to guess the intended referent (e.g., a parrot, a girl or a parrot-looking girl). This game was chosen because it is a popular, traditional game that was likely to elicit natural responses without requiring metalinguistic awareness (which has been found to hinder young children's performance in figurative language tasks; see Gibbs, 1994; Pouscoulous, 2011). 
Relatedly, the similes and metaphors used in this study were based on physical similarity, rather than psychological similarity (e.g., the parrot-looking girl had a nose that resembled the parrot's beak) because early studies have reported that metaphors that can be appreciated perceptually are easier to comprehend at a younger age. In a classic study, Asch and Nerlove (1960) showed that polysemous words such as 'hard', 'sweet' or 'bright' are first understood as physical properties, and it is not until age 11 that children appreciate their meaning as psychological properties (for a review of early studies, see Vosniadou, 1986). In order to make our task accessible to preschoolers, we used metaphors and similes that relied on physical similarities between people and animals.

We also followed Skordos and Papafragou (2016) in using a block design, such that simile trials always followed metaphor trials. This block design was intended to highlight the contrast between the categorization and comparison clues (e.g., 'Lucy is a parrot' vs 'Lucy is like a parrot') so that children more readily appreciated that the two expressions can function as alternatives. According to Skordos and Papafragou (2016), appreciating that the speaker is using one of two alternative terms is fundamental to the derivation of scalar implicatures.

The design of the guessing game required that children derived a scalar implicature in order to disambiguate the simile clue. When interpreted literally, 'Lucy is like a parrot' would be true of both the parrot and the parrot-looking girl (for experimental evidence with adults, see RubioFernandez et al., 2016). However, from a pragmatics point of view, such a description would only be maximally informative of the parrot-looking girl: if the Experimenter was referring to the parrot, she should have said 'Lucy is a parrot'. The fact that the Experimenter chose the weaker description would imply that the stronger one does not hold (i.e. it is not the case that Lucy is a parrot). Thus, if preschool children are able to derive the scalar implicature 'Lucy is not a parrot' 
when interpreting the simile 'Lucy is like a parrot', they should select the girl who looks like the parrot, rather than the parrot, even though both are similar to a parrot, literally speaking.

Regarding our experimental hypotheses, we predicted a literal bias in the metaphor condition across the preschool years (e.g., children would understand 'Lucy is a parrot' as a literal description of the parrot, rather than a metaphorical description of the parrot-looking girl), in line with early developmental studies on figurative language (Vosniadou, 1986, 1987a, 1987b, 1989). By contrast, in the simile condition, we predicted that literal responses would decrease with increasing age, revealing a developing ability to derive scalar implicatures.

\section{Experiment 1}

\section{Methods}

\section{Participants}

Ninety-nine children were recruited for the first experiment. The first three groups were recruited from preschool: 24 3-year-olds $(\mathrm{M}=3 ; 7$, range = 3;1-3;11, 15 girls), 18 4-year-olds $(\mathrm{M}$ $=4 ; 7$, range $=4 ; 3-5 ; 0,8$ girls $)$ and 18 5-year-olds $(M=5 ; 5$, range $=5 ; 1-5 ; 11,7$ girls $)$. The other two groups were recruited from primary and middle school, respectively: 19 6-year-olds $(\mathrm{M}=$ $6 ; 6$, range $=6 ; 0-7 ; 0,7$ girls $)$ and 2013 -year-olds $(M=13 ; 2$, range $=12 ; 6-14 ; 9,10$ girls $)$. All children were native Spanish speakers recruited from a school in Northern Spain which serves middle-class families. Ethical approval for the task was obtained from [the authors' institution] and minders of children in each grade were informed of the study. The number of children recruited was determined by the permissions that were obtained.

\section{Materials and design}


Six drawings consisting of 3 figures each were designed for the task and printed in individual booklets. An animal, a child (gender balanced) and an identical child with some feature resembling the animal were included in each drawing. The figure of the child that shared features with the other two figures was always placed in the middle in order to highlight the resemblances with the other two, and the positions of the other child and the animal were counterbalanced across trials. The animal features of the middle figures included: big frog-like eyes, pronounced monkey-like ears, a long giraffe-like neck, sharp shark-like teeth, messy lionlike hair and a curvy beak-like nose resembling a parrot. The six drawings were presented in the same gender-alternating order to all children. The first block of three trials was administered in the Metaphor condition (i.e. the clue was a categorization statement) and the second block in the Simile condition (i.e. the clue was a comparison statement).

\section{Procedure}

The Experimenter played the Spanish equivalent of the game 'I spy with my little eye' individually with children 3-6 years. The Experimenter started the game with the phrase 'I see, I see' (in Spanish: 'Veo, veo'), to which the child is supposed to answer 'What do you see?' ('¿Qué ves?’). The Experimenter then responded with a name and a clue: 'I see Lucy. Let me give you a clue: Lucy is a parrot' or 'Lucy is like a parrot', depending on the condition. The child then had to guess who Lucy was and point to one of the three figures, which the Experimenter marked on the paper.

The task was administered as a class exercise for the 13-year-olds, who were told that their responses would serve as control data for younger children. These children were given booklets with the drawings and for each drawing, the Experimenter gave the corresponding clue out loud and they marked their choice on the paper. The 13-year-olds were only tested in the Metaphor 
condition because they were expected to be at ceiling in the Simile condition, whereas a baseline was needed for children's appreciation of the ambiguity inherent in the metaphor trials (gloss: was Lucy a girl who looked like a parrot, or was she a parrot with a girl's name?).

Responses were coded as (a) Literal, (b) Pragmatic and (c) Neither. The label 'literal' referred to the non-figurative interpretation of the categorization statement (e.g., 'Lucy is a parrot' as referring to the parrot) and the logical interpretation of the comparison (e.g., 'Lucy is like a parrot' as referring to the parrot). The label 'pragmatic' referred to the figurative interpretation of the categorizations (e.g., 'Lucy is a parrot' as referring to the parrot-looking girl) and the scalar-implicature interpretation of the comparisons (e.g., 'Lucy is like a parrot' as referring to the parrot-looking girl, and not the parrot).

\section{Results and discussion}

Children's responses in the Metaphor and Simile conditions are plotted in Figures 2 and 3, respectively. Given the negligible percentages of Neither responses (M: 3\% in the Metaphor condition and $1 \%$ in the Simile condition), statistical analyses focused on Literal and Pragmatic responses. Overall, children's preference for Literal and Pragmatic responses were taken as evidence that they understood the comparison to the animal in both the Metaphor and Simile conditions, and therefore disregarded the dissimilar figure.

\section{INSERT FIG. 2 AROUND HERE}

Looking first at children's literal responses in the Metaphor condition, two-tailed binomial tests revealed a significant preference for the literal interpretation in children ages 3-6 years (all $\left.p^{\prime} \mathbf{s}<.001\right)$. By contrast, the 13-year-olds were at chance between the literal and pragmatic interpretations, suggesting that they appreciated the ambiguity between the two readings. 
Looking at the literal responses in the Simile condition, 3-year-olds were significantly above chance $(p<.001)$, revealing a reliable preference for the literal interpretation; 4-year-olds were at chance between the literal and pragmatic interpretations $(p=1.0)$, and 5- and 6-year-olds were below chance $(p<.001)$, showing a reliable preference for the pragmatic interpretation. These results suggest a developmental trend in pre-schoolers' derivation of scalar implicatures when interpreting similes.

We analyzed the data from the subset of children presented with both the Metaphor and Simile conditions (ages 3-6, $\mathrm{n}=79$ ) using logistic mixed effects regression in $\mathrm{R}$ (version 3.5.1; 2018). We modelled the outcome variable of interpretation ( $1=$ Literal, $0=$ Pragmatic), with Condition (Metaphor, Simile) and Age as fixed effects and Participants and Items as random effects. Deviation coding was used for Condition (Metaphor=-.5, Simile $=.5$ ), while participant age was entered as a scaled continuous predictor. The model was fitted with the maximal random effect structure (Barr et al., 2013). Results revealed a main effect of Condition ( $\beta=-3.918$, $p<.001)$ : more literal interpretations were observed in the Metaphor condition, and Age $(\beta=-$ 1.041, $p=.029$ ): literal interpretations decreased with increasing age (see Fig. 4 and Table 1 in the Supplementary Materials). There was also a Condition x Age interaction $(\beta=-3.596, p<.001)$ driven by a main effect of Age on Simile interpretation $(\beta=-3.913, p<.001)$ : literal interpretations of similes decreased with increasing age.

\section{- INSERT FIG. 4 AROUND HERE}

Metaphor data from the children presented with both conditions (ages 3-6, n=79) and data from children presented only with the Metaphor condition (age 13, $n=20$ ) were combined and analysed using logistic mixed effects regression. We modelled the outcome variable of 
interpretation (1=Literal, $0=$ Pragmatic) with Age (mean centred and scaled) as a fixed effect and Participants and Items as random effects, with the maximal random effect structure. Results revealed a main effect of Age $(\beta=-1.959, p=.017)$ : literal interpretations of metaphors decreased with increasing age (see Fig. 5 and Table 2 in the Supplementary Materials). INSERT FIG. 5 AROUND HERE

The results of the Metaphor condition replicated previous findings of a literal bias in young children, supporting our predictions: preschoolers (ages 3-5) and first graders (age 6) showed a reliable preference for the literal interpretation, whereas older children (age 13) appreciated the ambiguity in the categorization statements, giving a comparable number of literal and metaphorical responses. It must be noted that the clues used in this game were not contextualized and were therefore not a fair assessment of young children's abilities with figurative language (see Di Paola et al., 2019; Pouscoulous \& Tomasello, 2019). What can be concluded from these results, however, is that in the absence of context, it is not until after age 6 (and perhaps not even until middle school) that children appreciate the literal-metaphorical ambiguity inherent in the clues.

Regarding the Simile condition, there was an early preference for the literal interpretation that decreased with increasing age, also as predicted. The older children's preference for the character who looked like the animal, rather than the animal, might be explained as an effect of the names used in the clues - which were people's names, rather than animals'. However, all preschool children interpreted people's names as referring to the animal in the Metaphor condition, making it unlikely that their performance in the Simile condition was simply determined by the kind of name used in the clue. 
A more plausible, alternative interpretation of the developmental trend observed in the Simile condition is that it may have been accentuated by the metaphor trials administered in the first half of the task, which may have primed the literal response in the younger age groups (a sort of perseverance error), while highlighting the two alternative expressions for the older children (as intended). We therefore ran a follow-up experiment testing kids only in the simile condition to see if they would show an earlier preference for the pragmatic interpretation. We also used photographs, in addition to drawings, in case some of the young children in Experiment 1 had understood the drawings to be similar to the real animals they depicted (gloss: a drawing of a parrot is like a real parrot).

\section{Experiment 2}

\section{Methods}

\section{Participants}

Two-hundred and one individuals were recruited for the second experiment. They were divided into two groups: (a) children tested with drawings, including 27 3-year-olds $(\mathrm{M}=3 ; 7$, range $=2 ; 10-3 ; 11,14$ girls $), 314$-year-olds $(M=4 ; 5$, range $=4 ; 0-4 ; 10,17$ girls $)$ and 29 5-yearolds $(\mathrm{M}=5 ; 8$, range $=5 ; 0-6 ; 5,14$ girls $)$, and (b) individuals tested with photographs, including 29 3-year-olds (range $=2 ; 9-3 ; 8$, mean $=3 ; 2,17$ girls), 32 4-year-olds (range $=3 ; 9-4 ; 8$, mean $=$ 4;3, 10 girls), 35 5-year-olds (range $=4 ; 9-5 ; 8$, mean $=5 ; 3,20$ girls) and 18 adults (range $=20$ 23 , mean $=20 ; 8,10$ women). Children were recruited from the same preschool as in Experiment

1. Adults were Spanish university students contacted via email to volunteer as control subjects.

\section{Materials and procedure}


The same 6 drawings that were used in Experiment 1 were used again but the cues were all formulated as comparison statements (e.g., 'Lucy is like a parrot'). Parallel to the drawings, 5 sets of 3 photographs were put together including one photograph of an animal, one of a child and one of a similar child whose face was painted like the animal. The face paintings were of clearly recognizable animals selected through piloting: panda, tiger, cat, zebra and rabbit.

The testing procedure was the same one used in the Simile condition in Experiment 1. For the adults, a PDF was built with the slides used with the children. The clue was written above the photographs and the photographs were labelled A-B-C (see Fig. 6). Adult participants emailed their responses. Responses were coded the same as before.

INSERT FIG. 6 AROUND HERE

\section{Results and discussion}

Children's responses in the Drawing and Photograph conditions are plotted in Figures 7 and 8 , respectively. Given the negligible percentages of Neither responses $(M=6 \%$ in the Drawing condition and $1 \%$ in the Photograph condition), statistical analyses focused on Literal and Pragmatic responses.

INSERT FIG. 7 AROUND HERE

Looking first at children's literal responses in the Drawing condition, two-tailed binomial tests revealed a marginally reliable preference for the literal interpretation in 3 -year-olds $(p=.60)$ and a reliable rejection of the literal interpretation in 4 - and 5-year-olds $(p<.001)$. In the Photograph condition, all preschool groups were below chance, revealing a reliable preference for the pragmatic interpretation $(p<.001)$. Adults always chose the pragmatic interpretation (with the exception of a single response). 
Using logistic mixed effects regression, we modelled the outcome variable of interpretation (1=Literal, $0=$ Pragmatic) with Presentation (Drawing, Photograph) and Age as fixed effects and Participants and Items as random effects (with the maximal random effects structure). Deviation coding was used for Presentation (Drawing=-.5, Photograph=.5), while participant age was entered as a scaled continuous predictor. There was a main effect of Age $(\beta=-9.253, p<.001)$ : literal interpretations of similes decrease with increasing age (see Fig. 9 and Table 3 in the Supplementary Materials). There was also a trend towards a main effect of Presentation $(\beta=-2.574, p=.089)$, with fewer literal interpretations in the Photograph condition. However, the Age x Presentation interaction did not approach significance $(\beta=-.701, p=.874)$.

\section{INSERT FIG. 9 AROUND HERE}

Compared to Experiment 1, the results of Experiment 2 revealed a lower rate of literal interpretations in the younger age groups, suggesting that the first block of metaphor trials in Experiment 1 might have primed younger children to continue selecting the literal response in the simile trials. Using photographs instead of drawings also seemed to improve performance in the 3-year-olds, who went from a trend towards literal interpretations with drawings to a reliable preference for pragmatic interpretations with photographs. The better performance observed with photographs suggests that some 3-year-olds may have taken the drawings of the animals to be similar to the actual animals depicted. Such interpretation of the results, while clearly speculative, suggests a different type of literal interpretation of the simile clues (gloss: which of these drawings looks more like the real animal). Overall, preschoolers' derivation of scalar implicatures increased with age, with 5-year-olds showing adult-like performance when tested with photographs. 
We interpret children's performance with similes (e.g., 'Lucy is like a parrot') as evidence that they used a scalar implicature (i.e. 'Lucy is not a parrot') to choose between the animal and the child who resembled the animal (i.e. the parrot and the parrot-looking girl). However, we based this interpretation of our results on adult data. Rubio-Fernandez et al. (2017) observed that when adults were asked 'Is this animal like a tiger?' when presented with a picture of a tiger, they almost unanimously agreed, and report similar results in other experiments using a variety of materials. The question remains, however, as to whether the preschool children tested in this study also appreciated that both the parrot and the parrot-looking girl are like parrots, which would more strongly suggest that they derived a scalar implicature when selecting the parrot-looking girl. We investigated this question in the last experiment of the study.

\section{Experiment 3}

\section{Methods}

\section{Participants}

Forty-eight children were recruited for the third experiment: 163 -year-olds in their first year of preschool $(\mathrm{M}=3 ; 5$, range $=2 ; 9-3 ; 8,10$ girls $), 16$ 4-year-olds in their second year $(\mathrm{M}=4 ; 5$, range $=3 ; 10-4 ; 7,9$ girls $)$ and 165 -year-olds in their third and final year of preschool $(\mathrm{M}=5 ; 6$, range $=4 ; 9-5 ; 9,8$ girls). Children were recruited from the same preschool as in the first two experiments.

\section{Materials and procedure}

The same materials used in the Photograph version of Experiment 2 were used again in Experiment 3. However, rather than giving children a clue (e.g., 'Sonya is like a panda'), children were asked whether the animal or the child in the picture were like the relevant animal. 
For example, the Experimenter would point at the picture of a panda and ask the child 'Is this animal like a panda?' The Experimenter would then point at the picture of the panda-looking girl and ask the child 'Is this girl like a panda?', and repeat the question while pointing at the last picture of a girl (see Fig. 6). The Experimenter moved from left to right when pointing at the three pictures in each trial, and noted the children's responses on a score sheet.

\section{Results and discussion}

Children's responses ('Yes, it is like the animal' or 'No, it is not like the animal') are plotted in Figure 10. For each age group, positive responses were reliably above chance in the two critical conditions (Animal and Animal-looking Child), whereas they were significantly below chance in the filler condition (all $p$ 's $<.001$ ). The negative responses observed in the filler trials confirm that children were responding according to the perceived similarity of the pictures to the relevant animal, and not just agreeing with the Experimenter.

To test whether there were any differences in children's responses to the two critical conditions, we used logistic mixed effects regression, modelling the outcome variable of response $(1=$ Yes, $0=$ No) with Condition (Animal, Animal-looking Child) and Age as fixed effects and Participants and Items as random effects (with the maximal random effects structure). Deviation coding was used for Condition (Animal=-.5, Child=.5), while participant age was entered as a scaled continuous predictor. Results showed no main effect of Condition $(p=.755)$ or Age $(p=.748)$, nor was there a Condition $\mathrm{x}$ Age interaction $(p=.115)$ (see Fig. 10 and Table 4 in the Supplementary Materials). 
The results of Experiment 3 confirm that, like the adults in Rubio-Fernandez et al. (2017), preschool children between 3-5 years responded positively to the question 'Is this animal like a panda?' when asked about a panda, and also to the analogous question 'Is this girl like a panda?' when asked about a panda-looking girl, revealing no differences between critical conditions. Importantly, children responded negatively when asked about a girl who did not look like a panda, which confirms they were not simply agreeing with the Experimenter across the board. Children's appreciation that both the animal and the child were like the animal offers strong support for the interpretation that children as young as 3 years old derived a scalar implicature of the form ' $x$ is not a $y$ ' when selecting the child over the animal in the Simile condition of Experiments 1 and 2.

\section{General discussion}

Young children are known to find similes easier than metaphors (Reynolds \& Ortony, 1980; Vosniadou \& Ortony, 1983; Vosniadou et al., 1984; Seidenberg \& Bernstein, 1986; Happé, 1995). Here preschool and first-grade children (ages 3-6) showed a stronger preference for the literal interpretation of potentially metaphorical statements ('Lucy is a parrot') than for that of similes ('Lucy is like a parrot'), unlike their middle school counterparts (age 13) who appreciated the literal-metaphorical ambiguity. These results support the view that young children suffer from a literal bias when interpreting metaphors out of context, although their pragmatic abilities can be more sophisticated when figurative meanings are more accessible in the context (Pouscoulous, 2011).

Central to the aim of this study, preschool children's initial preference for the logical interpretation of similes decreased with increasing age, revealing a growing preference for the 
pragmatically enriched interpretation: if Lucy is said to be like a parrot, that means she is not a parrot (otherwise the clue would have been 'Lucy is a parrot'). When testing children with photographs (rather than drawings), even 3-year-olds showed a reliable preference for the pragmatic interpretation, while 5-year-olds' performance was comparable to that of adults. These results confirm that when tested with appropriate materials and protocols, even young preschool children are sensitive to scalar implicatures (see Pouscoulous et al., 2007; Stiller et al., 2015; Skordos \& Papafragou, 2016; Horowitz et al., 2018).

Recent metaphor studies employing more child-friendly procedures have also revealed improved performance in preschoolers (Rubio-Fernandez \& Grassmann, 2016; Di Paola et al., 2019; Pouscoulous \& Tomasello, 2019) compared to the metalingustic judgements that were required to succeed in early metaphor studies (see Vosniadou, 1986). In the present study, the enhanced pragmatic performance observed in the simile condition relative to the metaphor condition might seem to suggest that preschoolers are able to derive scalar implicatures before they can interpret metaphors. However, such an interpretation is likely to underestimate preschoolers' abilities with metaphorical language, which clearly improve when figurative uses are properly contextualized (see Falkum et al., 2017; Köder \& Falkum, 2019). Future studies should therefore compare preschoolers' pragmatic abilities when deriving scalar implicatures and interpreting metaphors using equally suitable tasks that prevent young children from defaulting to a literal bias in the absence of context.

The block design of Experiment 1 intended to contrast the two alternative expressions under investigation (e.g., 'Lucy is a parrot' vs 'Lucy is like a parrot'), potentially making the pragmatic interpretation of the similes more accessible (Skordos \& Papafragou, 2016). However, when the categorization-comparison scale was not highlighted in Experiment 2, preschoolers' performance 
was not compromised, suggesting scalar implicatures were highly accessible in this task. One methodological reason why this task may have been particularly easy for preschoolers is that, unlike earlier studies (e.g., Noveck, 2001; Papafragou \& Musolino, 2003; Barner et al., 2011), it did not require that underinformative descriptions be rejected as pragmatically infelicitous (e.g., children did not have to reject 'Luci is like a parrot' when predicated of a parrot). Instead, children had to select the character that better fit the description by favoring of a pragmatic interpretation over a logical reading (see also Stiller et al., 2015; Horowitz et al., 2018).

While sentence judgement tasks may be harder for preschoolers than reference disambiguation tasks, it should be noted that they are not necessarily a good test of children's abilities with scalar implicatures (for discussion, see Katsos \& Bishop, 2011). As for the present results, children seemed to appreciate that both the parrot and the parrot-looking girl were similar to a parrot (Experiment 3), but that the parrot-looking girl was the one who better fit the description 'Lucy is like a parrot' (Experiments 1 and 2). Future studies should try to establish at what age children start rejecting 'Lucy is like a parrot' as an underinformative description of an actual parrot.

A number of recent studies have also observed young preschoolers' comprehension of implicatures using arbitrary or ad hoc scales (e.g., inferring 'Fred did not eat the whole cupcake' from the utterance 'Fred ate the frosting'; Papafragou \& Tantalou, 2004; Stiller et al., 2015; Horowitz et al., 2018). Because these studies used child-friendly paradigms that did not require rejecting underinformative statements, and tried to make alternative expressions available at testing, it is difficult to determine whether the earlier age of success is related to the arbitrary nature of the scales, to the improved experimental methods, or to a combination of the two. While generally consistent with the hypothesis that young children struggle with scalar implicatures because of their limitations with generating alternatives (Barner et al., 2011), the results of 
Horowitz et al. (2018) suggest that partial quantifier knowledge is another factor that could explain why young children have trouble deriving scalar implicatures. This seems to suggest that young children may have less problems deriving implicatures from ad hoc scales because those rely on general informativity expectations (gloss: why specify that Fred ate the frosting if he actually ate the whole cupcake?), rather than the semantics of individual terms. Relatedly, Papafragou and Skordos (2016) have argued that the first step in being able to derive a scalar implicature is to learn the semantics of the individual terms, and then learn that these alternatives form a scale. This suggests that deriving implicatures with ad hoc scales may be easier for young children, as it relies entirely on pragmatic reasoning, and does not require acquiring specific semantic meanings. Future studies should directly compare young children's abilities with implicatures using lexicalized and ad hoc scales.

The preschool children in the present study were able to derive scalar implicatures at a younger age than in other studies, with 3-year-olds showing a reliable preference for the pragmatic interpretation in the photograph version of the task and 5-year-olds performing comparably to adults. A possible reason why young children are able to derive higher rates of scalar implicatures with similes than with other scalar expressions is that categorization and comparison statements are likely to be highly frequent in child-directed speech (e.g., 'A surgeon is a doctor' or 'Leopards are like cheetahs') and their acquisition may therefore happen earlier than that of quantifiers (e.g., 'some' vs 'all') or logical connectives (e.g., 'or' vs 'and'). Future research should therefore investigate children's exposure to different scalar terms and its effect on their ability to derive scalar implicatures. In the case of similes and metaphors (or comparisons and categorization statements, more generally) even children in the first year of preschool can show a reliable preference for the pragmatically enriched meaning, supporting the view that children's 
documented difficulties with scalar implicatures need not stem from their limited pragmatic abilities but from the protracted acquisition of alternative expressions and their scalarity.

\section{References}

Asch, S. E., \& Nerlove, H. (1960). The development of double function terms in children. Perspectives in Psychological Theory, 47-60.

Barnden, J. A. (2012). Metaphor and simile: Fallacies concerning comparison, ellipsis, and inter paraphrase. Metaphor and Symbol 27(4), 265-282.

Barner, D., Brooks, N., \& Bale, A. (2011). Accessing the unsaid: The role of scalar alternatives in children's pragmatic inference. Cognition, 118(1), 84-93.

Barr, D. J., Levy, R., Scheepers, C., \& Tily, H. J. (2013). Random effects structure for confirmatory hypothesis testing: Keep it maximal. Journal of Memory and Language, 68(3), $255-278$.

Bloom, P. (2002). How children learn the meanings of words. MIT press.

Braine, M. D., \& Rumain, B. (1981). Development of comprehension of “or": Evidence for a sequence of competencies. Journal of Experimental Child Psychology, 31(1), 46-70.

Carston, R. 2002. Thoughts and utterances: The pragmatics of explicit communication. Oxford: Blackwell Publishing.

Chierchia, G., Crain, S., Guasti, M. T., Gualmini, A., \& Meroni, L. (2001). The acquisition of disjunction: Evidence for a grammatical view of scalar implicatures. In Proceedings of the $25^{\text {th }}$ Boston University Conference on Language Development (pp. 157168). 
Clark, E. V. (1973). What's in a word? On the child's acquisition of semantics in his first language. In Cognitive development and acquisition of language (pp. 65-110). Academic Press.

Di Paola, S., Domaneschi, F., \& Pouscoulous, N. (2019). Metaphorical developing minds: The role of multiple factors in the development of metaphor comprehension. Journal of Pragmatics.

Falkum, I. L., Recasens, M., \& Clark, E. V. (2017). “The moustache sits down first": on the acquisition of metonymy. Journal of Child Language, 44(1), 87-119.

Gershkoff-Stowe, L., Connell, B., \& Smith, L. (2006). Priming overgeneralizations in two-and four-year-old children. Journal of Child Language, 33(3), 461-486.

Gibbs, R. W. (1994). The poetics of mind: Figurative thought, language and understanding. Cambridge: Cambridge University Press.

Glucksberg, S. (2011). Understanding metaphors: The paradox of unlike things compared. In Affective computing and sentiment analysis: Emotion, metaphor and terminology (pp. 1-12). New York, NY: Springer.

Glucksberg, S., \& Keysar, B. (1990). Understanding metaphorical comparisons: Beyond similarity. Psychological Review 97(1): 3-18.

Gualmini, A., Crain, S., Meroni, L., Chierchia, G., \& Guasti, M. T. (2001, October). At the semantics/pragmatics interface in child language. In Semantics and Linguistic Theory (Vol. 11, pp. 231-247).

Guasti, M. T., Chierchia, G., Crain, S., Foppolo, F., Gualmini, A., \& Meroni, L. (2005). Why children and adults sometimes (but not always) compute implicatures. Language and Cognitive Processes, 20(5), 667-696. 
Happé, F. G. (1995). Understanding minds and metaphors: Insights from the study of figurative language in autism. Metaphor and Symbol, 10(4), 275-295.

Horowitz, A. C., Schneider, R. M., \& Frank, M. C. (2018). The trouble with quantifiers: Exploring children's deficits in scalar implicature. Child development, 89(6), 572-593.

Hudson, J., \& Nelson, K. (1984). Play with language: Overextensions as analogies. Journal of Child Language, 11(2), 337-346.

Katsos, N., \& Bishop, D. V. (2011). Pragmatic tolerance: Implications for the acquisition of informativeness and implicature. Cognition, 120(1), 67-81.

Köder, F., \& Falkum, I. L. (2019). Children's metonymy comprehension: Evidence from eye-tracking and picture selection. Journal of Pragmatics.

Miller, K., Schmitt, C., Chang, H., \& Munn, A. (2005). Young children understand some implicatures. In Proceedings of the 29th Annual Boston University Conference on Language Development (pp. 389-400).

Noveck, I. A. (2001). When children are more logical than adults: Experimental investigations of scalar implicature. Cognition, 78(2), 165-188.

Ortony, A. (1979). Beyond literal similarity. Psychological Review 86(3): 161-180.

Papafragou, A., \& Musolino, J. (2003). Scalar implicatures: Experiments at the semantics/ pragmatics interface. Cognition, 86(3), 253-282.

Papafragou, A., \& Skordos, D. (2016). Scalar implicature. The Oxford Handbook of Developmental Linguistics (pp. 611-632). Oxford: Oxford Universtiy Press.

Papafragou, A., \& Tantalou, N. (2004). Children's computation of implicatures. Language Acquisition, 12, 71-82. 
Pouscoulous, N. (2011). Metaphor: For adults only? Belgian Journal of Linguistics, $25(1), 51-79$.

Pouscoulous, N., \& Tomasello, M. (2019). Early birds: Metaphor understanding in 3year-olds. Journal of Pragmatics.

Pouscoulous, N., Noveck, I. A., Politzer, G., \& Bastide, A. (2007). A developmental investigation of processing costs in implicature production. Language Acquisition, 14(4), 347 375 .

R Core Team (2018). R: A language and environment for statistical computing. R Foundation for Statistical Computing, Vienna, Austria. URL https:/www.R-project.org/.

Reynolds, R. E., \& Ortony, A. (1980). Some issues in the measurement of children's comprehension of metaphorical language. Child Development, 1110-1119.

Rubio-Fernandez, P., Geurts, B., \& Cummins, C. (2017). Is an apple like a fruit? A study on comparison and categorisation statements. Review of Philosophy and Psychology, 8(2), $367-390$.

Rubio-Fernandez, P., \& Grassmann, S. (2016). Metaphors as second labels: Difficult for preschool children? Journal of Psycholinguistic Research, 45(4), 931-944.

Seidenberg, P. L., \& Bernstein, D. K. (1986). The comprehension of similes and metaphors by learning disabled and non learning-disabled children. Language, Speech, and Hearing Services in Schools, 17(3), 219-229.

Skordos, D., \& Papafragou, A. (2016). Children's derivation of scalar implicatures: Alternatives and relevance. Cognition, 153, 6-18.

Smith, C. L. (1980). Quantifiers and question answering in young children. Journal of Experimental Child Psychology, 30(2), 191-205. 
Stiller, A. J., Goodman, N. D., \& Frank, M. C. (2015). Ad hoc implicature in preschool children. Language Learning and Development, 11, 176-190

Vosniadou, S. (1986). Children and metaphors. Center for the Study of Reading Technical Report; no. 370.

Vosniadou, S. (1987a). Children and metaphors. Child Development, 58(3), 870-885.

Vosniadou, S. (1987b). Contextual and linguistic factors in children's comprehension of nonliteral language. Metaphor and Symbol, 2(1), 1-11.

Vosniadou, S. (1989). Context and the development of metaphor comprehension. Metaphor and Symbolic Activity, 4, 159-171.

Vosniadou, S. (1995). Analogical reasoning in cognitive development. Metaphor and Symbol, 10(4), 297-308.

Vosniadou, S., \& Ortony, A. (1983). The emergence of the literal-metaphoricalanomalous distinction in young children. Child Development, 54(1), 154-161.

Vosniadou, S., Ortony, A., Reynolds, R., \&Wilson, P. (1984). Sources of difficulty in children's understanding of metaphorical language. Child Development, 55(4), 1588-1606.

Winner, E., McCarthy, M., Kleinman, S., \& Gardner, H. (1979). First metaphors. New Directions in Child Development, 3, 29-41.

Zharikov, S., \& Gentner, D. (2002). Why do metaphors seem deeper than similes? In Proceedings of the 24th annual meeting of the Cognitive Science Society (pp. 976-981). 


\section{Figures}

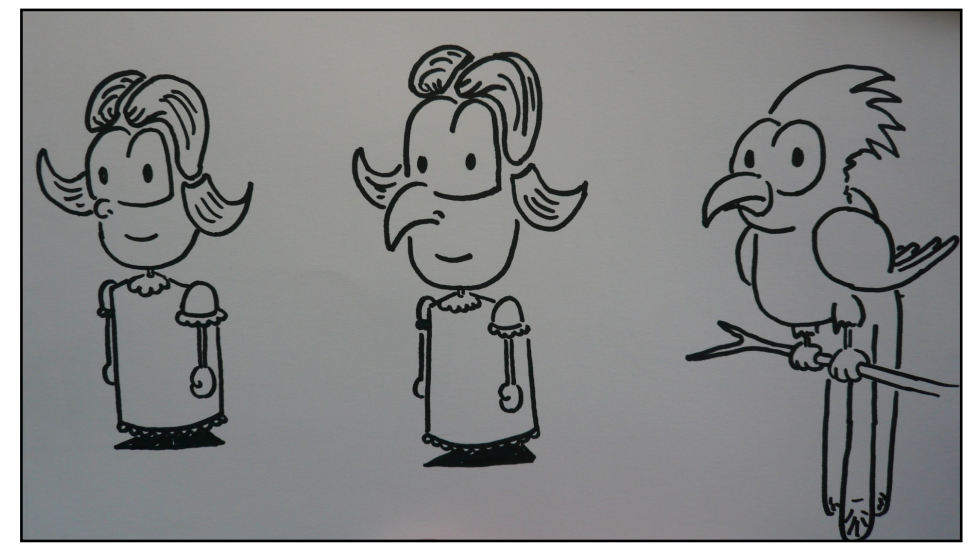

Fig. 1: Sample item for the game 'I spy with my little eye', in which the Experimenter gave the child a clue in the metaphor form ('Lucy is a parrot') or the simile form ('Lucy is like a parrot'). Rates of animal selections were used to measure a literal bias in the Metaphor condition and a logical bias in the Simile condition.

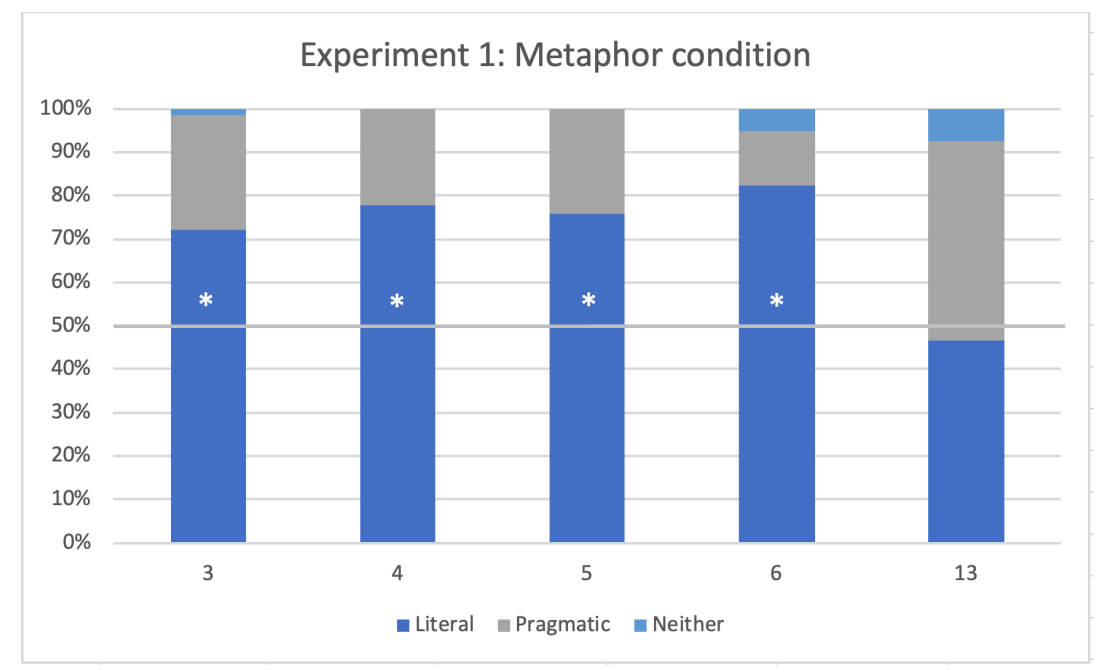

Fig. 2: Percentages of Literal, Pragmatic and Neither responses in the Metaphor condition (Trial block 1) for each age group. Asterisks indicate a reliable preference for the literal interpretation ( $p$ <.001). 


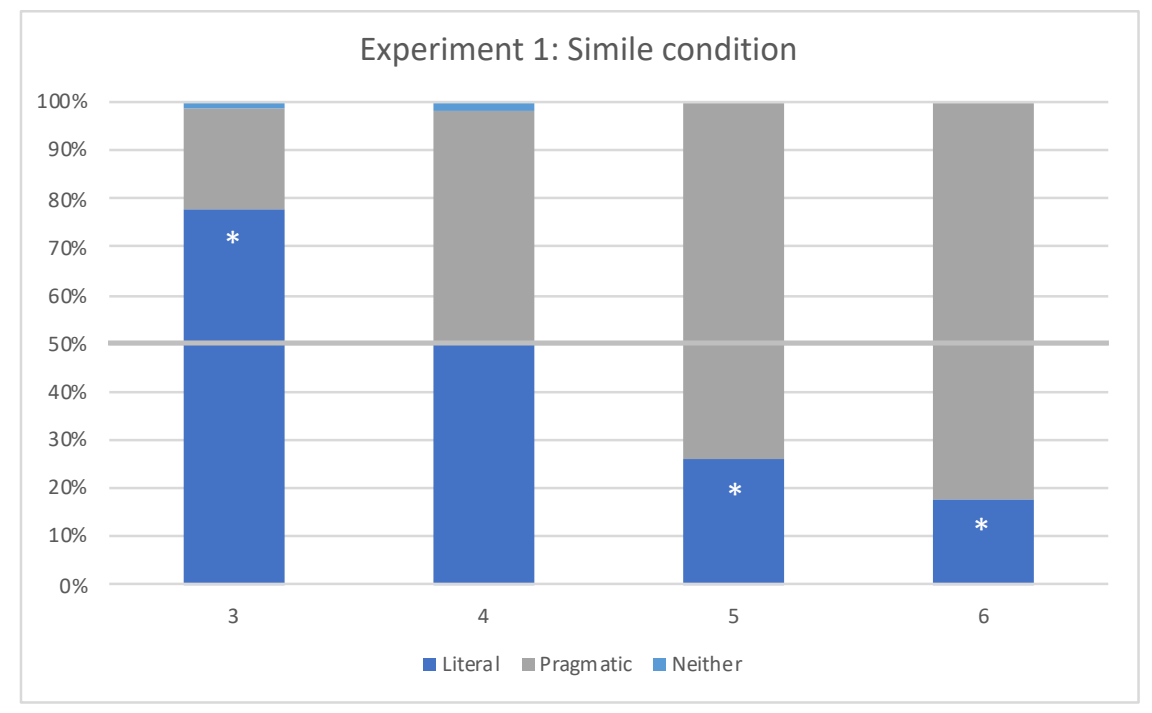

Fig. 3: Percentages of Literal, Pragmatic and Neither responses in the Simile condition (Trial block 2) for each age group. Asterisks indicate a reliable preference (above chance) or rejection (below chance) of the literal interpretation $(p<.001)$.

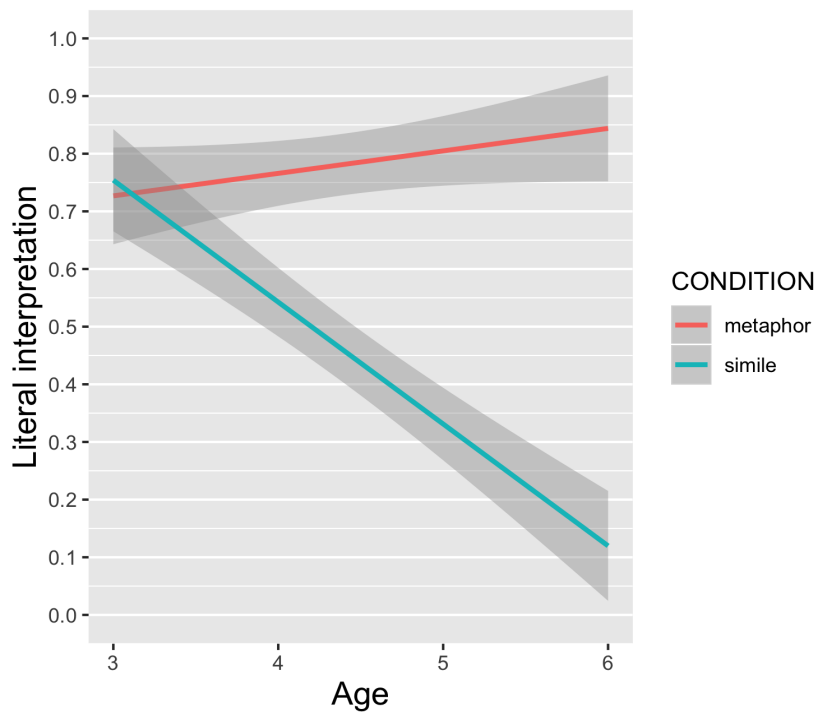

Fig. 4: Regression lines showing literal interpretations by age in the two conditions of Experiment 1. The shaded bands around the regression lines represent a $95 \%$ confidence region for the regression fit. 


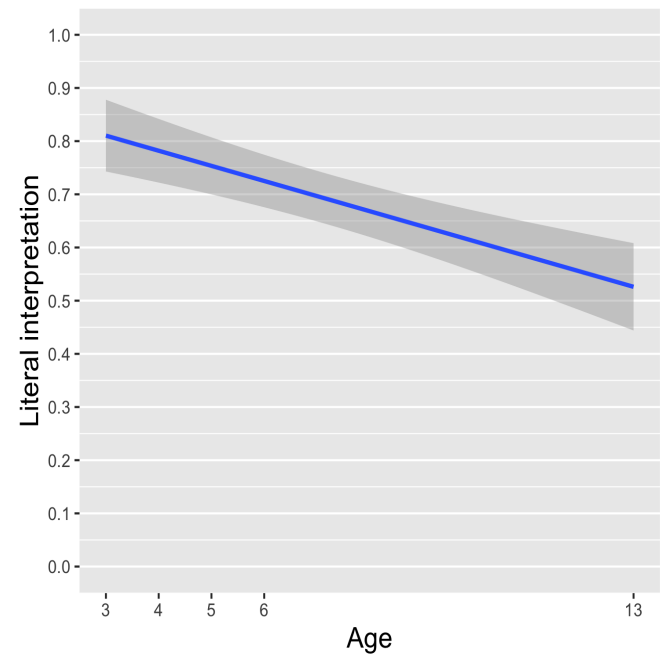

Fig. 5: Regression line showing literal interpretations for metaphors by age. The shaded band around the regression line represents a 95\% confidence region for the regression fit.

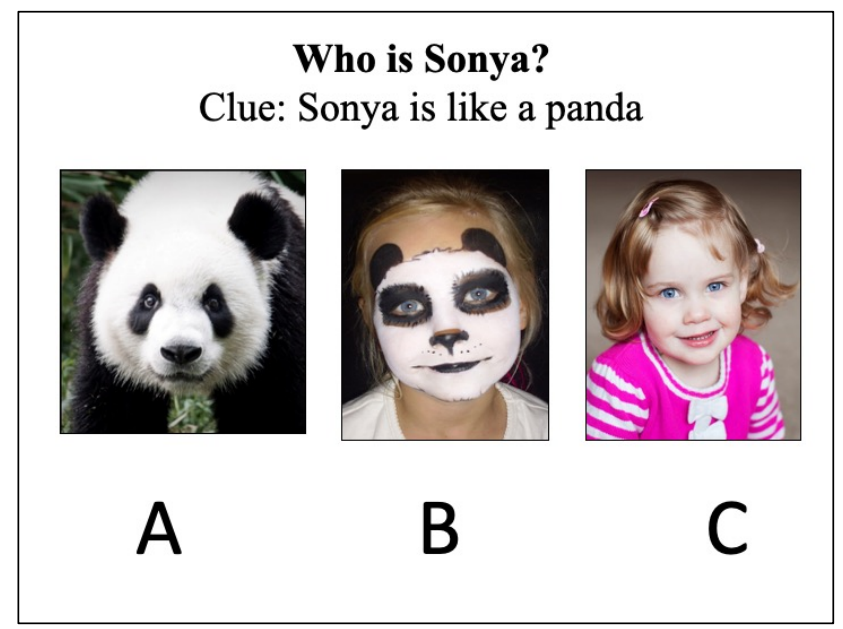

Fig. 6: Sample item from the photo version of the simile task as adapted for adults in Experiment 2. 


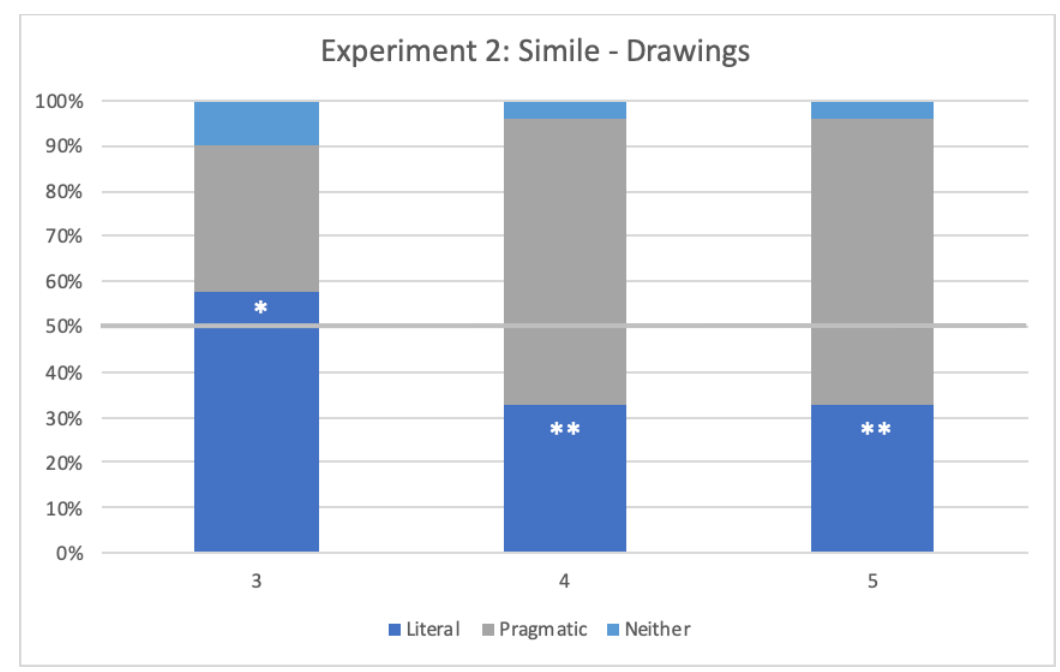

Fig. 7: Percentages of Literal, Pragmatic and Neither responses in the Drawings condition for each age group. Asterisks indicate a reliable preference (above chance) or rejection (below chance) of the literal interpretation (single asterisk; $p=.60$; two asterisks: $p<.001$ ).

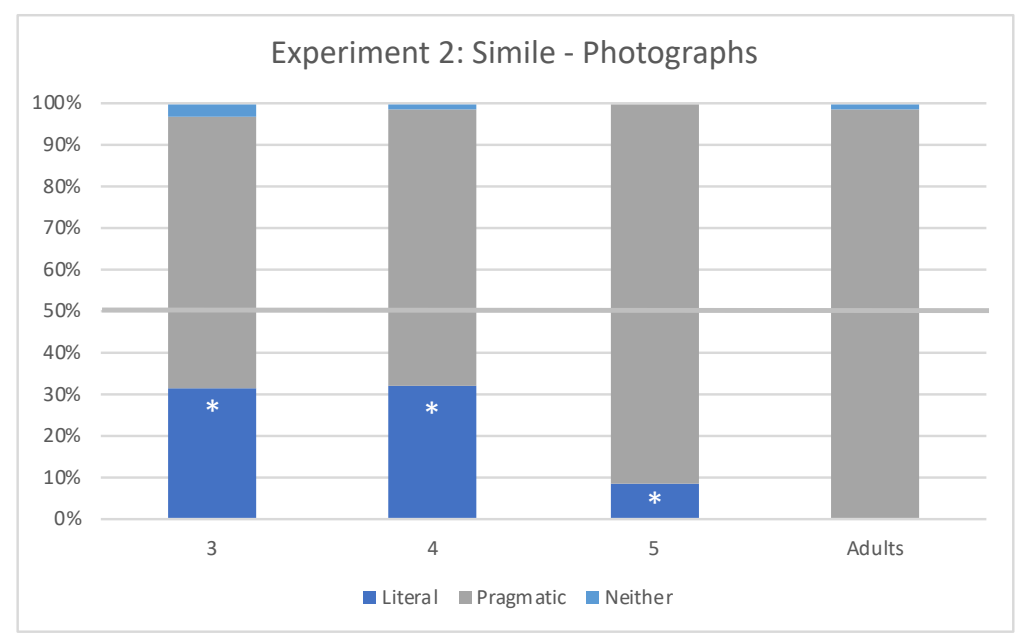

Fig. 8: Percentages of Literal, Pragmatic and Neither responses in the Photographs condition for each age group. Asterisks indicate a reliable rejection of the literal interpretation (below chance; $p$ $<.001)$. 


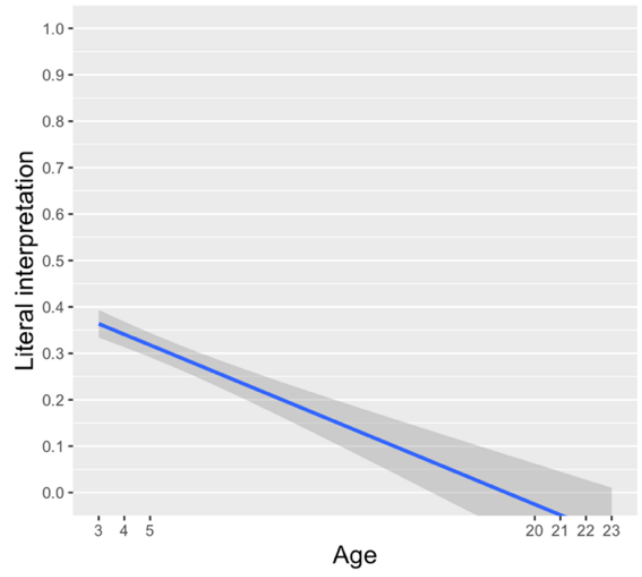

Fig. 9. Regression line showing literal interpretations of similes by age. The shaded band surrounding the regression line represents a 95\% confidence region for the regression fit.

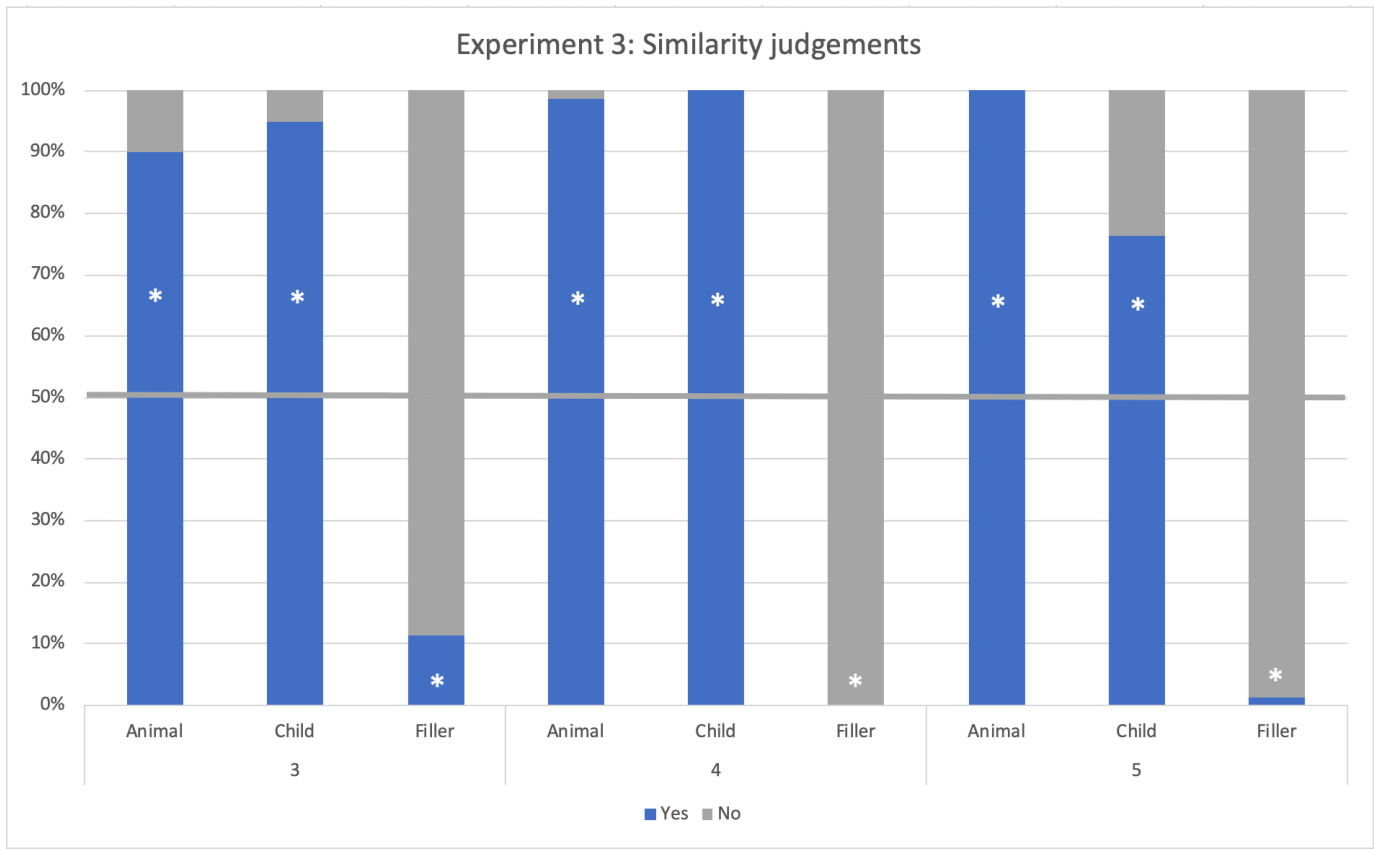

Fig. 10: Percentages of 'Yes' (it is like an $\mathrm{x}$ ) and 'No' (it is not like an $\mathrm{x}$ ) responses for each age group. Asterisks indicate a reliable preference for (above chance) or rejection of (below chance) the positive response $(p<.001)$. 